\title{
Some Analytic or Asymptotic Confluent Expansions for Functions of Several Variables
}

\author{
By H. M. Srivastava and Rekha Panda*
}

\begin{abstract}
This paper aims at presenting multivariable extensions of the recent results of J. L. Fields [5] and others (cf. [1]-[4]) on certain analytic or asymptotic confluent expansions for functions of one and two variables. It is also demonstrated how these extensions would apply, for instance, to derive an asymptotic confluent expansion for a certain class of the generalized Lauricella function of several variables.
\end{abstract}

1. Introduction. In recent years several writers have contributed to the theory of analytic or asymptotic confluent expansions for functions of one and two variables (see, for instance, [1]-[5] ; see also [6, pp. 50-57]). The object of the present paper is to discuss extensions of these results to certain functions of several variables. In order to illustrate the usefulness of these extensions, an asymptotic confluent expansion for a certain class of the generalized Lauricella function [7, p. 454 et seq.] is obtained. It is also shown how this last expansion (4.7) below can be further specialized to yield the corresponding asymptotic confluent expansion for the ordinary Lauricella functions $F_{A}^{(r)}, F_{B}^{(r)}$ and $F_{D}^{(r)}$ of $r$ variables.

The following Tricomi-Erdélyi result (cf., e.g., [6, p. 33]) will be required in our investigation:

$$
\begin{array}{r}
\frac{\Gamma(z+\alpha)}{\Gamma(z+\beta)}=z^{\alpha-\beta} \sum_{j=0}^{N-1} \frac{(-1)^{j}(\beta-\alpha)_{j}}{j !} B_{j}^{(\alpha-\beta+1)}(\alpha) z^{-j}+z^{\alpha-\beta} O\left(z^{-N}\right) \\
|\arg (z+\alpha)| \leqslant \pi-\epsilon, \epsilon>0
\end{array}
$$

where $\alpha$ and $\beta$ are bounded complex numbers, and $B_{j}^{(\alpha-\beta+1)}(\alpha)$ is the generalized Bernoulli polynomial.

2. Analytic confluent expansions. Our first result is the analytic confluent expansion (2.3) contained in

THEOREM 1. Let

$$
\sum_{k_{1}, \ldots, k_{r}=0}^{\infty} a\left(k_{1}, \ldots, k_{r}\right) z_{1}^{k_{1}} \ldots z_{r}^{k_{r}}<\infty, \quad\left|z_{i}\right|<R_{i}, i=1, \ldots, r
$$

Received November 11, 1974.

AMS (MOS) subject classifications (1970). Primary 33A30, 33A15; Secondary 41 A60.

Key words and phrases. Analytic confluent expansion, asymptotic confluent expansion, generalized Lauricella functions, generalized Bernoulli polynomials and numbers, Vandermonde's theorem, Poincaré coefficients, confluent hypergeometric function. A-7353.

* This work was supported in part by the National Research Council of Canada under grant

For a preliminary report on this work see Notices Amer. Math. Soc., v. 22, 1975, pp. A-11A-12, Abstract 75T-B9. 
Then $^{* *}$

$$
F\left[z_{i} ; \mu_{i} ; \sigma_{i}\right]=\sum_{k_{1}, \ldots, k_{r}=0}^{\infty} a\left(k_{1}, \ldots, k_{r}\right) \prod_{i=1}^{r} \frac{\left(\sigma_{i}\right)_{\mu_{i} k_{i}}}{\left(k_{i} !\right)^{\mu_{i}}}\left(\frac{z_{i}}{\sigma_{i}}\right)^{k_{i}}<\infty
$$

provided $\left|z_{i}\right| \sigma_{i} \mid<\mu_{i}^{-\mu_{i}} R_{i}, \mu_{i}>0, i=1, \ldots, r$; and $F\left(z_{1}, \ldots, z_{r}: \sigma\right)$ can be rearranged in the form,

$$
F\left(z_{1}, \ldots, z_{r}: \sigma\right)=\sum_{j_{1}, \ldots, j_{r}=0}^{\infty} g\left(j_{1}, \ldots, j_{r} ; z_{1}, \ldots, z_{r}\right) \sigma^{-j_{1}-\cdots-j_{r}},
$$

where

$$
\left|z_{i} / \sigma\right|<R_{i}, \quad i=1, \ldots, r, \quad F\left(z_{1}, \ldots, z_{r}: \sigma\right)=F\left[z_{i} ; 1 ; \sigma\right]
$$

and $g\left(j_{1}, \ldots, j_{r} ; z_{1}, \ldots, z_{r}\right)$ are given explicitly by (2.9) below.

Further, for $j_{1}, \ldots, j_{r} \geqslant 1$, the coefficients, $g\left(j_{1}, \ldots, j_{r} ; z_{1}, \ldots, z_{r}\right)$, can be expressed in terms of the derivatives of

$$
g\left(z_{1}, \ldots, z_{r}\right)=g\left(0, \ldots, 0 ; z_{1}, \ldots, z_{r}\right) .
$$

Proof. From (2.1) and the test for absolute convergence of multiple series given, for instance, by Srivastava and Daoust [8, p. 157 et seq.], it is easily verified that the series for $F\left[z_{i} ; \mu_{i} ; \sigma_{i}\right]$ in (2.2) converges absolutely when $\left|z_{i} / \sigma_{i}\right|<\mu_{i}^{-\mu_{i}} R_{i}, i=1, \ldots, r$.

For $\sigma \neq 0$, and integers $k_{i} \geqslant 0, i=1, \ldots, r$, we have

$$
\frac{(\sigma)_{k_{i}}}{\sigma^{k_{i}}}=\left(1+\frac{k_{i}}{\sigma}\right)^{-1} \prod_{j_{i}=0}^{k_{i}}\left(1+\frac{j_{i}}{\sigma}\right)=\sum_{j_{i}=0}^{k_{i}} \alpha_{i}\left(j_{i}, k_{i}\right) \sigma^{-j_{i}}, \text { say, }
$$

where $\alpha_{i}\left(j_{i}, k_{i}\right)>0, i=1, \ldots, r$.

Substitution in (2.2) with $\mu_{i}=1$ and $\sigma_{i}=1, i=1, \ldots, r$, will show that $F\left(z_{1}, \ldots, z_{r}: \sigma\right)$ is majorized by the convergent series,

$$
\begin{aligned}
\sum_{k_{1}, \ldots, k_{r}=0}^{\infty} & \frac{\left|a\left(k_{1}, \ldots, k_{r}\right)\right|\left|z_{1}\right|^{\left.k_{1} \ldots z_{r}\right|^{k}}}{k_{1} ! \cdots k_{r} !} \\
& \cdot \sum_{j_{1}=0}^{k_{1}} \cdots \sum_{j_{r}=0}^{k_{r}} \prod_{i=1}^{r}\left\{\alpha_{i}\left(j_{i}, k_{i}\right)\right\}|\sigma|^{-j_{1}-\cdots-j_{r}} \\
= & \sum_{k_{1}, \ldots, k_{r}=0}^{\infty} \frac{\left|a\left(k_{1}, \ldots, k_{r}\right)\right|(|\sigma|)_{k_{1}} \cdots(|\sigma|)_{k_{r}}}{k_{1} ! \cdots k_{r} !}\left|\frac{z_{1}}{\sigma}\right|^{k_{1}} \cdots\left|\frac{z_{r}}{\sigma}\right|^{k_{r}},
\end{aligned}
$$

where $\left|z_{i} / \sigma\right|<R_{i}, i=1, \ldots, r$.

Thus $F\left(z_{1}, \ldots, z_{r}: \sigma\right)$ can be rearranged in descending powers of $\sigma$. Moreover,

${ }^{* *}$ Throughout this paper we abbreviate, for convenien
$F\left[z_{1}, \ldots, z_{r} ; \mu_{1}, \ldots, \mu_{r} ; \sigma_{1}, \ldots, \sigma_{r}\right]$ by $F\left[z_{i} ; \mu_{i} ; \sigma_{i}\right]$ 
since $\sigma$ is arbitrary, the coefficients $g\left(j_{1}, \ldots, j_{r} ; z_{1}, \ldots, z_{r}\right)$ will converge for arbitrary $z_{1}, \ldots, z_{r}$.

In order to identify the $\alpha_{i}\left(j_{i}, k_{i}\right)$ in (2.5), we compare (2.5) and the TricomiErdélyi formula (1.1) with $z=\sigma, \alpha=k_{i}$ and $\beta=0$; we thus obtain

$$
\alpha_{i}\left(j_{i}, k_{i}\right)=\left(1-k_{i}\right)_{j_{i}} B_{j_{i}}^{\left(k_{i}\right)} / j_{i} !, \quad i=1, \ldots, r
$$

where $B_{n}^{(a)}=B_{n}^{(a)}(0)$ are the generalized Bernoulli numbers. Notice that

$$
\alpha_{i}\left(j_{i}, k_{i}\right)= \begin{cases}1, & \text { if } j_{i}=0, k_{i} \geqslant 0, \\ 0, & \text { if } j_{i} \geqslant 1, k_{i}=0 \text { or } k_{i}=1,\end{cases}
$$

for $i=1, \ldots, r$.

From (2.3), (2.5) and (2.7) it follows that

$$
\begin{aligned}
& g\left(j_{1}, \ldots, j_{r} ; z_{1}, \ldots, z_{r}\right) \\
& \quad=\sum_{k_{1}, \ldots, k_{r}=0}^{\infty} a\left(k_{1}, \ldots, k_{r}\right) \prod_{i=1}^{r} \frac{\left(1-k_{i}\right)_{j_{i}} z_{i}^{k_{i}}}{j_{i} ! k_{i} !} B_{j_{i}}^{\left(k_{i}\right)} .
\end{aligned}
$$

For $k_{i}, j_{i} \geqslant 1, \quad k_{i}^{-1} B_{j_{i}}^{\left(k_{i}\right)}$ is a polynomial of degree $\left(j_{i}-1\right)$ in $k_{i}$. Thus, if $\Delta_{x}$ denotes the forward difference operator with respect to $x$, i.e., $\Delta_{x}\{f(x)\}=f(x+1)-$ $f(x)$, we have for $j_{i} \geqslant 1, i=1, \ldots, r$,

$$
B_{j_{i}}^{\left(k_{i}\right)}=k_{i} \sum_{m_{i}=0}^{j_{i}-1} \frac{(-1)^{m_{i}\left(1+j_{i}-k_{i}\right)_{m_{i}}}}{m_{i} !} \phi\left(m_{i}, j_{i}\right),
$$

where, for convenience,

$$
\phi\left(m_{i}, j_{i}\right)=\left[\Delta_{k_{i}}^{m_{i}}\left\{B_{j_{i}}^{\left(k_{i}\right)} / k_{i}\right\}\right]_{k_{i}=j_{i}+1} \quad(i=1, \ldots, r) .
$$

In view of (2.4), (2.9) and (2.10), it follows fairly easily that for $j_{i} \geqslant 1, i=1, \ldots, r$,

$$
\begin{aligned}
g\left(j_{1}, \ldots, j_{r} ; z_{1}, \ldots, z_{r}\right) & \sum_{m_{1}=0}^{j_{1}-1} \cdots \sum_{m_{r}=0}^{j_{r}-1}(-1)^{J} \frac{\partial^{r+J+m}}{\partial z_{1}^{1+j_{1}+m_{1}} \ldots \partial z_{r}^{1+j_{r}+m_{r}}} \\
& \left\{g\left(z_{1}, \ldots, z_{r}\right)\right\} \prod_{i=1}^{r} \frac{z_{i}^{1+j_{i}+m_{i}} \phi\left(m_{i}, j_{i}\right)}{j_{i} ! m_{i} !}
\end{aligned}
$$

where the $\phi\left(m_{i}, j_{i}\right)$ are given by $(2.11)$, and

$$
J=j_{1}+\cdots+j_{r}, \quad M=m_{1}+\cdots+m_{r} .
$$

This evidently completes the proof of Theorem 1 .

Remark 1. A markedly different form of Theorem 1 may be given, for instance, by replacing the product,

$$
\prod_{i=1}^{r} \frac{\left(\sigma_{i}\right)_{\mu_{i} k_{i}}}{\left(k_{i} !\right)^{\mu_{i}}}\left(\frac{z_{i}}{\sigma_{i}}\right)^{k_{i}}
$$


in (2.2) by

Remark 2. If we put

$$
(\sigma)_{\mu_{1} k_{1}}+\cdots+\mu_{r} k_{r} \prod_{i=1}^{r} \frac{\left(z_{i} / \sigma\right)^{k_{i}}}{\left(k_{i} !\right)^{\mu_{i}}}, \quad \mu_{i}>0
$$

$$
A_{m_{1}, \ldots, m_{r}}\left(z_{1}, \ldots, z_{r}\right)=z_{1}^{m_{1}} \ldots z_{r}^{m_{r}} \frac{\partial^{m_{1}+\cdots+m_{r}}}{\partial z_{1}^{m_{1}} \ldots \partial z_{r}^{m_{r}}}\left\{g\left(z_{1}, \ldots, z_{r}\right)\right\},
$$

where

$$
g\left(z_{1}, \ldots, z_{r}\right)=g\left(0, \ldots, 0 ; z_{1}, \ldots, z_{r}\right)
$$

$$
=\sum_{k_{1}, \ldots, k_{r}=0}^{\infty} a\left(k_{1}, \ldots, k_{r}\right) \frac{z_{1}^{k_{1}}}{k_{1} !} \cdots \frac{z_{r}^{k_{r}}}{k_{r} !},
$$

then the first few coefficients $g\left(j_{1}, \ldots, j_{r} ; z_{1}, \ldots, z_{r}\right)$, given by (2.9) and (2.12), are as follows:

$$
\begin{aligned}
& g\left(1,0, \ldots, 0 ; z_{1}, \ldots, z_{r}\right)=\frac{1}{2} A_{2,0, \ldots, 0}\left(z_{1}, \ldots, z_{r}\right), \\
& g\left(0, \ldots, 0,1 ; z_{1}, \ldots, z_{r}\right)=\frac{1}{2} A_{0, \ldots, 0,2}\left(z_{1}, \ldots, z_{r}\right) ; \\
& g\left(1,1,0, \ldots, 0 ; z_{1}, \ldots, z_{r}\right)=\frac{1}{4} A_{2,2,0, \ldots, 0}\left(z_{1}, \ldots, z_{r}\right), \\
& g\left(0, \ldots, 0,1,1 ; z_{1}, \ldots, z_{r}\right)=\frac{1}{4} A_{0, \ldots, 0,2,2}\left(z_{1}, \ldots, z_{r}\right), \\
& g\left(1, \ldots, 1 ; z_{1}, \ldots, z_{r}\right)=\frac{1}{2^{r}} A_{2, \ldots, 2}\left(z_{1}, \ldots, z_{r}\right) \\
& g\left(2,0, \ldots, 0 ; z_{1}, \ldots, z_{r}\right) \\
& =\frac{1}{3} A_{3,0, \ldots, 0}\left(z_{1}, \ldots, z_{r}\right)+\frac{1}{8} A_{4,0, \ldots, 0}\left(z_{1}, \ldots, z_{r}\right), \\
& g\left(0, \ldots, 0,2 ; z_{1}, \ldots, z_{r}\right) \\
& =\frac{1}{3} A_{0, \ldots, 0,3}\left(z_{1}, \ldots, z_{r}\right)+\frac{1}{8} A_{0, \ldots, 0,4}\left(z_{1}, \ldots, z_{r}\right) ;
\end{aligned}
$$$$
g\left(1,2,0, \ldots, 0 ; z_{1}, \ldots, z_{r}\right)
$$$$
=\frac{1}{6} A_{2,3,0, \ldots, 0}\left(z_{1}, \ldots, z_{r}\right)+\frac{1}{16} A_{2,4,0, \ldots, 0}\left(z_{1}, \ldots, z_{r}\right)
$$$$
g\left(2,1,0, \ldots, 0 ; z_{1}, \ldots, z_{r}\right)
$$$$
=\frac{1}{6} A_{3,2,0, \ldots, 0}\left(z_{1}, \ldots, z_{r}\right)+\frac{1}{16} A_{4,2,0, \ldots, 0}\left(z_{1}, \ldots, z_{r}\right) ;
$$ 


$$
\begin{aligned}
g\left(3,0, \ldots, 0 ; z_{1}, \ldots, z_{r}\right)= & \frac{1}{4} A_{4,0, \ldots, 0}\left(z_{1}, \ldots, z_{r}\right) \\
& +\frac{1}{6} A_{5,0, \ldots, 0}\left(z_{1}, \ldots, z_{r}\right)+\frac{1}{48} A_{6,0, \ldots, 0}\left(z_{1}, \ldots, z_{r}\right),
\end{aligned}
$$

et cetera.

Another analytic confluent expansion for functions of several variables is given by THEOREM 2. Let

$$
\sum_{k_{1}, \ldots, k_{r}=0}^{\infty} b\left(k_{1}, \ldots, k_{r}\right) z_{1}^{k_{1}} \ldots z_{r}^{k_{r}}<\infty, \quad\left|z_{i}\right|<R_{i}, i=1, \ldots, r .
$$

Then

$$
\begin{aligned}
& G\left[z_{i} ; \lambda_{i} ; \mu_{i} ; \nu_{i} ; \rho_{i}\right]= \sum_{k_{1}, \ldots, k_{r}=0}^{\infty} b\left(k_{1}, \ldots, k_{r}\right) \\
& \cdot \prod_{i=1}^{r} \frac{\left(-v_{i}\right)_{\mu_{i} k_{i}}\left(\nu_{i}+\lambda_{i}\right) \rho_{i} k_{i}}{\left(k_{i} !\right)^{\mu_{i}+\rho_{i}}}\left[-\frac{z_{i}}{v_{i}\left(\nu_{i}+\lambda_{i}\right)}\right]^{k_{i}}<\infty,
\end{aligned}
$$

provided $\left|z_{i}\right|<\left|\nu_{i}\left(\nu_{i}+\lambda_{i}\right)\right| \mu_{i}^{-\mu_{i}} \rho_{i}^{-\rho_{i}} R_{i}, \mu_{i}>0, \rho_{i}>0, i=1, \ldots, r ;$ and $G\left(z_{1}, \ldots, z_{r}: \nu, \lambda\right)$ can be rearranged in descending powers of $\nu(\nu+\lambda)$ to yield

$$
G\left(z_{1}, \ldots, z_{r}: \nu, \lambda\right)
$$

$$
=\sum_{j_{1}, \ldots, j_{r}=0}^{\infty} h\left(j_{1}, \ldots, j_{r} ; z_{1}, \ldots, z_{r} ; \lambda\right)[-\nu(\nu+\lambda)]^{-j_{1}-\cdots-j_{r}},
$$

where $\left|z_{i}\right|<|\nu(\nu+\lambda)| R_{i}, i=1, \ldots, r$,

$$
G\left(z_{1}, \ldots, z_{r}: \nu, \lambda\right)=G\left[z_{i} ; \lambda ; 1 ; \nu ; 1\right],
$$

and $h\left(j_{1}, \ldots, j_{r} ; z_{1}, \ldots, z_{r} ; \lambda\right)$ are given explicitly by (2.21) below.

Further, for $j_{1}, \ldots, j_{r} \geqslant 1$, the coefficients, $h\left(j_{1}, \ldots, j_{r} ; z_{1}, \ldots, z_{r} ; \lambda\right)$, can be expressed in terms of the derivatives of

$$
h\left(z_{1}, \ldots, z_{r}\right)=h\left(0, \ldots, 0 ; z_{1}, \ldots, z_{r} ; \lambda\right) .
$$

The proof of this theorem would run parallel to that of Theorem 1, and we omit details. Indeed, it is easily verified that

(2.21) $h\left(j_{1}, \ldots, j_{r} ; z_{1}, \ldots, z_{r} ; \lambda\right)=\sum_{k_{1}, \ldots, k_{r}=0}^{\infty} b\left(k_{1}, \ldots, k_{r}\right) \prod_{i=1}^{r} c_{j_{i}, k_{i}}(\lambda) \frac{z_{i}^{k_{i}}}{\left(k_{i} !\right)^{2}}$,

where

$$
\left\{\begin{array}{l}
c_{j_{i}+1, k_{i}}(\lambda)=\sum_{m_{i}=0}^{k_{i}-1} m_{i}\left(m_{i}+\lambda\right) c_{j_{i}, m_{i}}(\lambda), \\
c_{0, m_{i}}(\lambda)=1, \quad m_{i} \geqslant 0, \\
c_{j_{i}+1,0}(\lambda)=0, \quad i=1, \ldots, r .
\end{array}\right.
$$


If we write

$$
B_{m_{1}, \ldots, m_{r}}\left(z_{1}, \ldots, z_{r}\right)=z_{1}^{m_{1}} \ldots z_{r}^{m_{r}} \frac{\partial^{m_{1}+\cdots+m_{r}}}{\partial z_{1}^{m_{1}} \cdots \partial z_{r}^{m_{r}}}\left\{h\left(z_{1}, \ldots, z_{r}\right)\right\}
$$

where

$$
\begin{aligned}
h\left(z_{1}, \ldots, z_{r}\right) & =h\left(0, \ldots, 0 ; z_{1}, \ldots, z_{r} ; \lambda\right) \\
& =\sum_{k_{1}, \ldots, k_{r}=0}^{\infty} b\left(k_{1}, \ldots, k_{r}\right) \prod_{i=1}^{r} \frac{z_{i}^{k_{i}}}{\left(k_{i} !\right)^{2}}
\end{aligned}
$$

then we have the first few coefficients given by

$$
\begin{aligned}
& h\left(1,0, \ldots, 0 ; z_{1}, \ldots, z_{r} ; \lambda\right)=\frac{1}{3} B_{3,0, \ldots, 0}\left(z_{1}, \ldots, z_{r}\right) \\
& +\frac{1}{2}(\lambda+1) B_{2,0, \ldots, 0}\left(z_{1}, \ldots, z_{r}\right), \\
& h\left(0, \ldots, 0,1 ; z_{1}, \ldots, z_{r} ; \lambda\right)=\frac{1}{3} B_{0, \ldots, 0,3}\left(z_{1}, \ldots, z_{r}\right) \\
& +\frac{1}{2}(\lambda+1) B_{0, \ldots, 0,2}\left(z_{1}, \ldots, z_{r}\right) \\
& h\left(2,0, \ldots, 0 ; z_{1}, \ldots, z_{r} ; \lambda\right)=\frac{1}{18} B_{6,0, \ldots, 0}\left(z_{1}, \ldots, z_{r}\right) \\
& +\frac{1}{30}(5 \lambda+17) B_{5,0, \ldots, 0}\left(z_{1}, \ldots, z_{r}\right) \\
& +\frac{1}{8}\left(\lambda^{2}+8 \lambda+11\right) B_{4,0, \ldots, 0}\left(z_{1}, \ldots, z_{r}\right) \\
& +\frac{1}{3}(\lambda+1)(\lambda+2) B_{3,0, \ldots, 0}\left(z_{1}, \ldots, z_{r}\right), \\
& h\left(0, \ldots, 0,2 ; z_{1}, \ldots, z_{r} ; \lambda\right)=\frac{1}{18} B_{0, \ldots, 0,6}\left(z_{1}, \ldots, z_{r}\right) \\
& +\frac{1}{30}(5 \lambda+17) B_{0, \ldots, 0,5}\left(z_{1}, \ldots, z_{r}\right)+\frac{1}{8}\left(\lambda^{2}+8 \lambda+11\right) B_{0, \ldots, 0,4}\left(z_{1}, \ldots, z_{r}\right) \\
& +\frac{1}{3}(\lambda+1)(\lambda+2) B_{0, \ldots, 0,3}\left(z_{1}, \ldots, z_{r}\right)
\end{aligned}
$$

et cetera.

Remark 3. A substantial variation of Theorem 2 would follow if, for instance, we replace the product,

$$
\prod_{i=1}^{r} \frac{\left(-v_{i}\right)_{\mu_{i} k_{i}}\left(\nu_{i}+\lambda_{i}\right)_{\rho_{i} k_{i}}}{\left(k_{i} !\right)^{\mu_{i}+\rho_{i}}}\left[-\frac{z_{i}}{v_{i}\left(\nu_{i}+\lambda_{i}\right)}\right]^{k_{i}}
$$


in $(2.17)$ by

$$
(-\nu)_{\mu_{1} k_{1}+\cdots+\mu_{r} k_{r}}(\nu+\lambda)_{\rho_{1} k_{1}}+\cdots+\rho_{r} k_{r} \prod_{i=1}^{r} \frac{\left[-z_{i} / \nu(\nu+\lambda)\right]^{k_{i}}}{\left(k_{i} !\right)^{\mu_{i}+\rho_{i}}},
$$

where, as before, $\mu_{i}, \rho_{i}>0, i=1, \ldots, r$.

Remark 4. If we let

$$
b\left(k_{1}, \ldots, k_{r}\right)=k_{1} ! \cdots k_{r} ! a\left(k_{1}, \ldots, k_{r}\right), \quad k_{i} \geqslant 0, i=1, \ldots, r,
$$

then the following relationship holds between Theorems 1 and 2:

$$
\lim _{\lambda \rightarrow \infty} G\left(z_{1}, \ldots, z_{r}: \nu, \lambda\right)=F\left(z_{1}, \ldots, z_{r}:-\nu\right),
$$

which is easy to verify.

3. Asymptotic confluent expansions. Our first result on asymptotic confluent expansions for functions of several variables is contained in

THEOREM 3. Let

$$
\sum_{k_{1}, \ldots, k_{r}=0}^{\infty} c\left(k_{1}, \ldots, k_{r}\right) z_{1}^{k_{1}} \ldots z_{r}^{k_{r}}<\infty, \quad\left|z_{i}\right|<R_{i}, i=1, \ldots, r .
$$

Then the series

$$
H\left[z_{i} ; \beta_{i} ; \mu_{i}\right]=\sum_{k_{1}, \ldots, k_{r}=0}^{\infty} c\left(k_{1}, \ldots, k_{r}\right) \prod_{i=1}^{r} \frac{\left(\beta_{i} z_{i}\right)^{k_{i}}}{k_{i} !\left(\beta_{i}\right)_{\mu_{i} k_{i}}}
$$

converges for all $z_{i}, \beta_{i} \neq 0,-1,-2, \ldots, \mu_{i}>0, i=1, \ldots, r$; and the function,

$$
H\left(z_{1}, \ldots, z_{r}: \beta\right)=H\left[z_{i} ; \beta ; 1\right],
$$

possesses the asymptotic confluent expansion,

$$
\begin{aligned}
H\left(z_{1}, \ldots, z_{r}: \beta\right) \sim & \sum_{j_{1}, \ldots, j_{r}=0}^{\infty} f\left(j_{1}, \ldots, j_{r} ; z_{1}, \ldots, z_{r}\right)(-\beta)^{-j_{1}-\cdots-j_{r}}, \\
& |\beta| \rightarrow \infty,|\arg (\beta)| \leqslant \pi-\epsilon, 0<\epsilon \leqslant \pi / 2, \\
& \left|z_{i}\right|<R_{i}, i=1, \ldots, r, \text { if }|\arg (\beta)| \leqslant \pi / 2 ; \\
& \left|z_{i}\right|<|\sin \arg (\beta)| R_{i}, i=1, \ldots, r, \text { if } \pi / 2 \leqslant|\arg (\beta)| \leqslant \pi-\epsilon,
\end{aligned}
$$

where the coefficients $f\left(j_{1}, \ldots, j_{r} ; z_{1}, \ldots, z_{r}\right)$ are given explicitly by (3.6) below.

Further, for $j_{1}, \ldots, j_{r} \geqslant 1$, these coefficients can be expressed in terms of the derivatives of

$$
f\left(z_{1}, \ldots, z_{r}\right)=f\left(0, \ldots, 0 ; z_{1}, \ldots, z_{r}\right) .
$$

Proof. The first part of this theorem stems from (3.1) and the test for convergence of multiple series [8, p. 157 et seq. $]$. The demonstration of the rest would make use of the Tricomi-Erdélyi formula (1.1) in a straightforward manner. Thus it is easily seen that 


$$
\begin{aligned}
f\left(j_{1}, \ldots, j_{r} ; z_{1}, \ldots, z_{r}\right) & \\
& =\sum_{k_{1}, \ldots, k_{r}=0}^{\infty} c\left(k_{1}, \ldots, k_{r}\right) \prod_{i=1}^{r} \frac{\left(k_{i}\right)_{j_{i}} z_{i}^{k_{i}}}{j_{i} !} B_{j_{i}}^{\left(1-k_{i}\right)},
\end{aligned}
$$

and for $j_{1}, \ldots, j_{r} \geqslant 1$, we have

$$
\begin{aligned}
f\left(j_{1}, \ldots, j_{r} ; z_{1}, \ldots, z_{r}\right)= & \sum_{m_{1}=0}^{2 j_{1}-2} \cdots \sum_{m_{r}=0}^{2 j_{r}-2} \prod_{i=1}^{r} \frac{z_{i}^{2+m_{i}} \psi\left(m_{i}\right)}{j_{i} ! m_{i} !} \\
& \cdot \frac{\partial^{2 r+m_{1}+\cdots+m_{r}}}{\partial z_{1}^{2+m_{1}} \ldots \partial z_{r}^{2+m_{r}}}\left\{f\left(z_{1}, \ldots, z_{r}\right)\right\},
\end{aligned}
$$

where, for convenience,

$$
\psi\left(m_{i}\right)=\left[\Delta_{k_{i}}^{m_{i}}\left\{\left(k_{i}\right)_{j_{i}} B_{j_{i}}^{\left(1-k_{i}\right)} /\left[k_{i}\left(k_{i}-1\right)\right]\right\}\right]_{k_{i}=2} \quad(i=1, \ldots, r) .
$$

Remark 5. A slightly different form of Theorem 3 may be given by replacing the product,

$$
\prod_{i=1}^{r} \frac{\left(\beta_{i} z_{i}\right)^{k_{i}}}{k_{i} !\left(\beta_{i}\right)_{\mu_{i} k_{i}}}
$$

in (3.2) by

$$
\left\{(\beta)_{\mu_{1} k_{1}+\cdots+\mu_{r} k_{r}}\right\}^{-1} \prod_{i=1}^{r} \frac{\left(\beta z_{i}\right)^{k_{i}}}{k_{i} !}, \quad \mu_{i}>0, i=1, \ldots, r .
$$

Remark 6. In terms of the derivatives,

$$
C_{m_{1}, \ldots, m_{r}}\left(z_{1}, \ldots, z_{r}\right)=z_{1}^{m_{1}} \ldots z_{r}^{m_{r}} \frac{\partial^{m_{1}+\cdots+m_{r}}}{\partial z_{1}^{m_{1}} \ldots \partial z_{r}^{m_{r}}}\left\{f\left(z_{1}, \ldots, z_{r}\right)\right\}
$$

where

$$
\begin{aligned}
f\left(z_{1}, \ldots, z_{r}\right) & =f\left(0, \ldots, 0 ; z_{1}, \ldots, z_{r}\right) \\
& =\sum_{k_{1}, \ldots, k_{r}=0}^{\infty} c\left(k_{1}, \ldots, k_{r}\right) z_{1}^{k_{1}} \ldots z_{r}^{k_{r}},
\end{aligned}
$$

the first few $f\left(j_{1}, \ldots, j_{r} ; z_{1}, \ldots, z_{r}\right)$ are given as follows:

$$
\begin{aligned}
& f\left(1,0, \ldots, 0 ; z_{1}, \ldots, z_{r}\right)=\frac{1}{2} C_{2,0, \ldots, 0}\left(z_{1}, \ldots, z_{r}\right) \\
& \vdots \\
& f\left(0, \ldots, 0,1 ; z_{1}, \ldots, z_{r}\right)=\frac{1}{2} C_{0, \ldots, 0,2}\left(z_{1}, \ldots, z_{r}\right) \\
& f\left(1, \ldots, 1 ; z_{1}, \ldots, z_{r}\right)=\frac{1}{2^{r}} C_{2, \ldots, 2}\left(z_{1}, \ldots, z_{r}\right) ;
\end{aligned}
$$




$$
\begin{aligned}
& f\left(2,0, \ldots, 0 ; z_{1}, \ldots, z_{r}\right)=\frac{1}{2} C_{2,0, \ldots, 0}\left(z_{1}, \ldots, z_{r}\right) \\
& +\frac{2}{3} C_{3,0, \ldots, 0}\left(z_{1}, \ldots, z_{r}\right)+\frac{1}{8} C_{4,0, \ldots, 0}\left(z_{1}, \ldots, z_{r}\right), \\
& \begin{aligned}
f\left(0, \ldots, 0,2 ; z_{1}, \ldots, z_{r}\right)= \\
\quad
\end{aligned} \\
& \quad+\frac{2}{3} C_{0, \ldots, 0,3}\left(z_{1}, \ldots, z_{r}\right)+\frac{1}{8} C_{0, \ldots, 0,4}\left(z_{1}, \ldots, z_{r}\right) ;
\end{aligned}
$$

et cetera.

For a nonnegative integer $n$, the multiple series defining the functions $F\left(z_{1}, \ldots, z_{r}:-n\right)$ and $G\left(z_{1}, \ldots, z_{r}: n, \lambda\right)$ in Theorems 1 and 2 would terminate and so these functions are well defined everywhere. Thus, under considerably weaker conditions than those stated earlier, Theorems 1 and 2 yield the asymptotic confluent expansions of $F\left(z_{1}, \ldots, z_{r}:-n\right)$ and $G\left(z_{1}, \ldots, z_{r}: n, \lambda\right)$, respectively, as $n \rightarrow \infty$. These immediate consequences of Theorems 1 and 2 are contained in

THEOREM 4. Let

$$
\begin{aligned}
& \sum_{k_{1}, \ldots, k_{r}=0}^{\infty} a\left(k_{1}, \ldots, k_{r}\right) \frac{z_{1}^{k_{1}}}{k_{1} !} \cdots \frac{z_{r}^{k_{r}}}{k_{r} !}<\infty, \quad\left|z_{i}\right|<S_{i}, i=1, \ldots, r \\
& \sum_{k_{1}, \ldots, k_{r}=0}^{\infty} b\left(k_{1}, \ldots, k_{r}\right) \frac{z_{1}^{k_{1}}}{\left(k_{1} !\right)^{2}} \cdots \frac{z_{r}^{k_{r}}}{\left(k_{r} !\right)^{2}}<\infty, \quad\left|z_{i}\right|<T_{i}, i=1, \ldots, r .
\end{aligned}
$$

Then, for an integer $n \geqslant 0$,

$$
F\left(z_{1}, \ldots, z_{r}:-n\right)=\sum_{k_{1}, \ldots, k_{r}=0}^{n} a\left(k_{1}, \ldots, k_{r}\right) \prod_{i=1}^{r} \frac{(-n)_{k_{i}}}{k_{i} !}\left(-\frac{z_{i}}{n}\right)^{k_{i}}
$$

$$
\begin{array}{r}
\sim \sum_{j_{1}, \ldots, j_{r}=0}^{\infty} g\left(j_{1}, \ldots, j_{r} ; z_{1}, \ldots, z_{r}\right)(-n)^{-j_{1}-\cdots-j_{r}}, \\
n \rightarrow \infty,\left|z_{i}\right|<S_{i}, i=1, \ldots, r,
\end{array}
$$

and

$$
\begin{aligned}
& G\left(z_{1}, \ldots, z_{r}: n, \lambda\right) \\
&= \sum_{k_{1}, \ldots, k_{r}=0}^{n} b\left(k_{1}, \ldots, k_{r}\right) \prod_{i=1}^{r} \frac{(-n)_{k_{i}}(n+\lambda)_{k_{i}}}{\left(k_{i} !\right)^{2}}\left[-\frac{z_{i}}{n(n+\lambda)}\right]^{k_{i}} \\
& \sim \sum_{j_{1}, \ldots, j_{r}=0}^{\infty} h\left(j_{1}, \ldots, j_{r} ; z_{1}, \ldots, z_{r} ; \lambda\right)[-n(n+\lambda)]^{-j_{1}-\cdots-j_{r}}, \\
& n \rightarrow \infty,\left|z_{i}\right|<T_{i}, i=1, \ldots, r,
\end{aligned}
$$

where the coefficients $g\left(j_{1}, \ldots, j_{r} ; z_{1}, \ldots, z_{r}\right)$ and $h\left(j_{1}, \ldots, j_{r} ; z_{1}, \ldots, z_{r} ; \lambda\right)$ are given explicitly by (2.9) and (2.21), respectively. 
The following theorem provides yet another interesting asymptotic confluent expansion for a function of several variables.

THEOREM 5. Let

$$
\begin{aligned}
U\left(z_{1}, \ldots, z_{r}\right)=\sum_{k_{1}, \ldots, k_{r}=0}^{\infty} d\left(k_{1}, \ldots, k_{r}\right) z_{1}^{k_{1}} \ldots z_{r}^{k_{r}}<\infty, \\
\\
\quad\left|z_{i}\right|<R_{i}, i=1, \ldots, r .
\end{aligned}
$$

Then

(3.16) $V\left[z_{i} ; \mu_{i} ; \sigma_{i} ; a_{i} ; b_{i}\right]=\sum_{k_{1}, \ldots, k_{r}=0}^{\infty} d\left(k_{1}, \ldots, k_{r}\right) \prod_{i=1}^{r} \frac{\left(\sigma_{i}+a_{i}\right)_{\mu_{i} k_{i}} z_{i}^{k_{i}}}{\left(\sigma_{i}+b_{i}\right)_{\mu_{i} k_{i}}}<\infty$, provided $\left|z_{i}\right|<R_{i}, \mu_{i}>0, \sigma_{i}+b_{i} \neq 0,-1,-2, \ldots, i=1, \ldots, r$; and for $\mu_{1}=\cdots=\mu_{r}=1$, it can be rearranged when $\left|z_{i}\right|<R_{i} / 2, i=1, \ldots, r$, to yield the expansion,

$$
\begin{aligned}
V & {\left[z_{i} ; 1 ; \sigma_{i} ; a_{i} ; b_{i}\right] } \\
& =\sum_{j_{1}, \ldots, j_{r}=0}^{\infty} w\left(j_{1}, \ldots, j_{r} ; z_{1}, \ldots, z_{r}\right)\left[\left(\sigma_{1}+b_{1}\right)_{j_{1}} \cdots\left(\sigma_{r}+b_{r}\right)_{j_{r}}\right]^{-1},
\end{aligned}
$$

where the coefficients $w\left(j_{1}, \ldots, j_{r} ; z_{1}, \ldots, z_{r}\right)$ are given explicitly by (3.21) below.

However, if the $a_{i}$ and $b_{i}$ are bounded, then (3.17) with $\sigma_{1}=\cdots=\sigma_{r}=\sigma$ results in the asymptotic expansion,

$$
\begin{aligned}
& V\left(z_{1}, \ldots, z_{r}: \sigma\right)=V\left[z_{i} ; 1 ; \sigma ; a_{i} ; b_{i}\right] \\
& \sim \sum_{j_{1}, \ldots, j_{r}=0}^{\infty} w\left(j_{1}, \ldots, j_{r} ; z_{1}, \ldots, z_{r}\right)\left[\left(\sigma+b_{1}\right)_{j_{1}} \cdots\left(\sigma+b_{r}\right)_{j_{r}}\right]^{-1}, \\
& |\sigma| \rightarrow \infty,\left|\arg \left(\sigma+b_{i}\right)\right| \leqslant \pi-\delta_{i}, \delta_{i}>0,\left|z_{i}\right|<R_{i}, i=1, \ldots, r .
\end{aligned}
$$

Moreover, if $\left[\left(\sigma+b_{1}\right)_{j_{1}} \cdots\left(\sigma+b_{r}\right)_{j_{r}}\right]^{-1}$ is expanded in powers of $1 / \sigma$, (3.18) can be written as an asymptotic confluent expansion in $1 / \sigma$.

Proof. The first assertion (3.16) would follow fairly readily as in Theorems 1-4. Also, since $\sigma_{i}+b_{i} \neq 0,-1,-2, \ldots, i=1, \ldots, r$, from the familiar Vandermonde Theorem we obtain

$$
\sum_{j_{i}=0}^{k_{i}} \frac{\left(-k_{i}\right)_{j_{i}}\left(b_{i}-a_{i}\right)_{j_{i}}}{j_{i} !\left(\sigma_{i}+b_{i}\right)_{j_{i}}}=\frac{\left(\sigma_{i}+a_{i}\right)_{k_{i}}}{\left(\sigma_{i}+b_{i}\right)_{k_{i}}}, \quad k_{i} \geqslant 0, i=1, \ldots, r .
$$

Making use of (3.15), we have

$$
\begin{aligned}
\frac{\partial^{j_{1}+\cdots+j_{r}}}{\partial z_{1}^{j_{1}} \ldots \partial z_{r}^{j_{r}}} & \left\{U\left(z_{1}, \ldots, z_{r}\right)\right\} \\
& =\sum_{k_{1}, \ldots, k_{r}=0}^{\infty} d\left(k_{1}, \ldots, k_{r}\right) \prod_{i=1}^{r}(-1)^{j_{i}}\left(-k_{i}\right)_{j_{i}} z_{i}^{k_{i}-j_{i}}
\end{aligned}
$$


which, in conjunction with (3.17) and (3.19), yields

$$
\begin{aligned}
& w\left(j_{1}, \ldots, j_{r} ; z_{1}, \ldots, z_{r}\right) \\
& =\prod_{i=1}^{r}\left\{\frac{\left(b_{i}-a_{i}\right)_{i_{i}}\left(-z_{i}\right)^{j_{i}}}{j_{i} !}\right\} \frac{\partial^{j_{1}+\cdots+j_{r}}}{\partial z_{1}^{j_{1}} \cdots \partial z_{r}^{j_{r}}}\left\{U\left(z_{1}, \ldots, z_{r}\right)\right\} .
\end{aligned}
$$

If we assume that $\left|z_{i}\right|<X_{i}<R_{i}, i=1, \ldots, r$, then the hypothesis (3.15) would evidently imply that

$$
d\left(k_{1}, \ldots, k_{r}\right)=O\left(X_{1}^{-k_{1}} \ldots X_{r}^{-k_{r}}\right), \quad k_{1}, \ldots, k_{r} \rightarrow \infty .
$$

From (3.17), (3.20) and (3.21) we thus observe that, for some constant $M>0$,

$$
\begin{aligned}
\left|V\left[z_{i} ; 1 ; \sigma_{i} ; a_{i} ; b_{i}\right]\right| \leqslant M \sum_{j_{1}, \ldots, j_{r}=0}^{\infty}\left|\prod_{i=1}^{r} \frac{\left(b_{i}-a_{i}\right)_{j_{i}}}{\left(\sigma_{i}+b_{i}\right)_{j_{i}}}\right| \\
\cdot \sum_{k_{1}, \ldots, k_{r}=0}^{\infty} \prod_{i=1}^{r}\left(\begin{array}{c}
k_{i}+j_{i} \\
j_{i}
\end{array}\right)\left(\frac{\left|z_{i}\right|}{X_{i}}\right)^{k_{i}+j_{i}} \\
=\left(1-\left|z_{1}\right| / X_{1}\right)^{-1} \ldots\left(1-\left|z_{r}\right| / X_{r}\right)^{-1} M \\
\quad \cdot \sum_{j_{1}, \ldots, j_{r}=0}^{\infty}\left|\prod_{i=1}^{r} \frac{\left(b_{i}-a_{i}\right)_{j_{i}}}{\left(\sigma_{i}+b_{i}\right)_{j_{i}}}\right|\left(\frac{X_{1}}{\left|z_{1}\right|}-1\right)^{-j_{1}} \cdots\left(\frac{X_{r}}{\left|z_{r}\right|}-1\right)^{-j_{r}},
\end{aligned}
$$

which obviously converges when

$$
\left(X_{i} /\left|z_{i}\right|-1\right)^{-1}<1, \quad i=1, \ldots, r
$$

that is, when

$$
\left|z_{i}\right|<X_{i} / 2<R_{i} / 2, \quad i=1, \ldots, r
$$

and the final assertion would follow from the fact that the $w\left(j_{1}, \ldots, j_{r} ; z_{1}, \ldots, z_{r}\right)$ in (3.18) are identifiable as the Poincaré coefficients of $V\left(z_{1}, \ldots, z_{r}: \sigma\right)$ as $|\sigma| \rightarrow \infty$.

Remark 7. Another version of Theorem 5 can be given by further multiplying the product,

$$
\prod_{i=1}^{r} \frac{\left(\sigma_{i}+a_{i}\right)_{\mu_{i} k_{i}} z_{i}^{k_{i}}}{\left(\sigma_{i}+b_{i}\right)_{\mu_{i} k_{i}}}
$$

in (3.16) by such quotients as

$$
(\sigma+a)_{\rho_{1} k_{1}}+\cdots+\rho_{r} k_{r} /(\sigma+b)_{\rho_{1} k_{1}}+\cdots+\rho_{r} k_{r}
$$

times $z_{j}^{K}$, where $K=\Sigma_{i=1}^{r} k_{i}-k_{j}, 1 \leqslant j \leqslant r, \sigma+b \neq 0,-1,-2, \ldots$, and $\rho_{i}>0$, $i=1, \ldots, r$.

In particular, for $\mu_{i}=\rho_{i}=1, \sigma_{i}=\sigma, i=1, \ldots, r$, and $j=1$, we may state

THEOREM 6. Let the hypothesis (3.15) of Theorem 5 be replaced by 


$$
W\left(z_{1}, \ldots, z_{r}\right)=\sum_{k_{1}, \ldots, k_{r}=0}^{\infty} d\left(k_{1}, \ldots, k_{r}\right) z_{1}^{k_{1}}\left(z_{1} z_{2}\right)^{k_{2}} \cdots\left(z_{1} z_{r}\right)^{k_{r}}<\infty
$$

where $\left|z_{1}\right|<R_{1}$ and $\left|z_{1} z_{j}\right|<R_{j}, j=2, \ldots, r$.

Then the series

$$
\begin{aligned}
& Y\left(z_{1}, \ldots, z_{r}: \sigma\right) \\
& =\sum_{k_{1}, \ldots, k_{r}=0}^{\infty} d\left(k_{1}, \ldots, k_{r}\right) \frac{\left(\sigma+a_{1}\right)_{k_{1}}+\cdots+k_{r}}{\left(\sigma+b_{1}\right)_{k_{1}}+\cdots+k_{r}} \frac{\Pi_{j=2}^{r}\left(\sigma+a_{j}\right)_{k_{j}}}{\Pi_{j=2}^{r}\left(\sigma+b_{j}\right)_{k_{j}}} \\
& \cdot z_{1}^{k_{1}}\left(z_{1} z_{2}\right)^{k_{2}} \cdots\left(z_{1} z_{r}\right)^{k_{r}} \text {, }
\end{aligned}
$$

converges for $\left|z_{1}\right|<R_{1},\left|z_{1} z_{j}\right|<R_{j}, j=2, \ldots, r, \sigma+b_{i} \neq 0,-1,-2, \ldots, i=$ $1, \ldots, r$; and it can be rearranged when $\left|z_{1}\right|<\epsilon_{1} R_{1},\left|z_{1} z_{j}\right|<\epsilon_{j} R_{j}, j=2, \ldots, r$, for some $\epsilon_{i}, 0<\epsilon_{i}<1, i=1, \ldots, r$, to yield the expansion,

$$
\begin{aligned}
& Y\left(z_{1}, \ldots, z_{r}: \sigma\right) \\
& \quad=\sum_{j_{1}, \ldots, j_{r}=0}^{\infty} u\left(j_{1}, \ldots, j_{r} ; z_{1}, \ldots, z_{r}\right)\left[\left(\sigma+b_{1}\right)_{j_{1}} \cdots\left(\sigma+b_{r}\right)_{j_{r}}\right]^{-1},
\end{aligned}
$$

where

$$
\begin{aligned}
& u\left(j_{1}, \ldots, j_{r} ; z_{1}, \ldots, z_{r}\right) \\
& \quad=\prod_{i=1}^{r}\left\{\frac{\left(b_{i}-a_{i}\right)_{j_{i}}\left(-z_{i}\right)^{j_{i}}}{j_{i} !}\right\} \frac{\partial^{j_{1}+\cdots+j_{r}}}{\partial z_{1}^{j_{1}} \cdots \partial z_{r}^{j_{r}}}\left\{W\left(z_{1}, \ldots, z_{r}\right)\right\} .
\end{aligned}
$$

Moreover, for bounded $a_{i}$ and $b_{i},(3.26)$ holds asymptotically as

$$
Y\left(z_{1}, \ldots, z_{r}: \sigma\right)
$$

$$
\sim \sum_{j_{1}, \ldots, j_{r}=0}^{\infty} u\left(j_{1}, \ldots, j_{r} ; z_{1}, \ldots, z_{r}\right)\left[\left(\sigma+b_{1}\right)_{j_{1}} \cdots\left(\sigma+b_{r}\right)_{j_{r}}\right]^{-1},
$$

$|\sigma| \rightarrow \infty,\left|\arg \left(\sigma+b_{i}\right)\right| \leqslant \pi-\delta_{i}, \delta_{i}>0, i=1, \ldots, r,\left|z_{1}\right|<R_{1},\left|z_{1} z_{j}\right|<R_{j}$, $j=2, \ldots, r$, which leads to an asymptotic confluent expansion in $1 / \sigma$ if $\left[\left(\sigma+b_{1}\right)_{j_{1}} \cdots\left(\sigma+b_{r}\right)_{j_{r}}\right]^{-1}$ is expanded in reciprocal powers of $\sigma$.

Remark 8. For $r=2$, this last theorem would evidently reduce to the main result (Theorem 1, p. 605) in Deshpande's paper [4].

4. Applications. By assigning suitable values to the arbitrary coefficients $a\left(k_{1}, \ldots, k_{r}\right), b\left(k_{1}, \ldots, k_{r}\right), c\left(k_{1}, \ldots, k_{r}\right)$ and $d\left(k_{1}, \ldots, k_{r}\right), k_{i} \geqslant 0, i=1$, $\ldots, r$, Theorems 1-6 can be applied to deduce analytic or asymptotic confluent expansions for various special functions of several variables; e.g., the Lauricella hypergeometric functions $F_{A}^{(r)}, F_{B}^{(r)}, F_{C}^{(r)}$ and $F_{D}^{(r)}$ of $r$ variables and their generalizations studied by Srivastava and Daoust ([7], [8]). As an illustration, we apply Theorems 1 and 4 to derive an asymptotic confluent expansion for a certain generalization of the Lauricella functions, defined by (cf. [7, p. 454]; see also [8, p. 158]) 


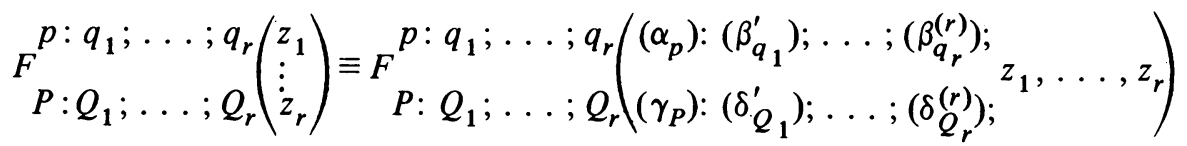

$$
\begin{aligned}
& =\sum_{k_{1}, \ldots, k_{r}=0}^{\infty} \Lambda\left(k_{1}, \ldots, k_{r}\right) \prod_{i=1}^{r} \frac{z_{i}^{k_{i}}}{k_{i} !},
\end{aligned}
$$

where, and in what follows,

$$
\Lambda\left(k_{1}^{0}, \ldots, k_{r}\right)=\frac{\Pi_{j=1}^{p}\left(\alpha_{j}\right)_{k_{1}}+\cdots+k_{r} \Pi_{j=1}^{q_{1}}\left(\beta_{j}^{\prime}\right)_{k_{1}} \cdots \Pi_{j=1}^{q_{r}}\left(\beta_{j}^{(r)}\right)_{k_{r}}}{\Pi_{j=1}^{P}\left(\gamma_{j}\right)_{k_{1}}+\cdots+k_{r} \Pi_{j=1}^{Q_{1}}\left(\delta_{j}^{\prime}\right)_{k_{1}} \cdots \Pi_{j=1}^{Q_{r}}\left(\delta_{j}^{(r)}\right)_{k_{r}}},
$$

and for convergence of the multiple series in (4.1),

$$
1+P+Q_{i}-p-q_{i} \geqslant 0, \quad i=1, \ldots, r
$$

the equality holds when, in addition,

$$
p>P \text { and }\left|z_{1}\right|^{1 /(p-P)}+\cdots+\left|z_{r}\right|^{1 /(p-P)}<1,
$$

or

$$
p \leqslant P \text { and } \max \left\{\left|z_{1}\right|, \ldots,\left|z_{r}\right|\right\}<1
$$

Thus, if we put

$$
\begin{aligned}
& D_{m_{1}, \ldots, m_{r}}\left(z_{1}, \ldots, z_{r}\right)=z_{1}^{m_{1}} \ldots z_{r}^{m_{r}} \Lambda\left(m_{1}, \ldots, m_{r}\right) \\
& \quad \begin{array}{l}
\quad: q_{1} ; \ldots ; q_{r}\left(\begin{array}{l}
\left(\alpha_{p}\right)+m_{1}+\cdots+m_{r}:\left(\beta_{q_{1}}^{\prime}\right)+m_{1} ; \ldots ;\left(\beta_{q_{r}}^{(r)}\right)+m_{r} ; \\
z_{1}, \ldots, z_{r}
\end{array}\right), \\
\quad P: Q_{1} ; \ldots ; Q_{r}\left(\gamma_{P}\right)+m_{1}+\cdots+m_{r}:\left(\delta_{Q_{1}}^{\prime}\right)+m_{1} ; \ldots ;\left(\delta_{Q_{r}}^{(r)}\right)+m_{r} ;
\end{array}
\end{aligned}
$$

and let $n$ be a nonnegative integer, we obtain the desired asymptotic expansion,

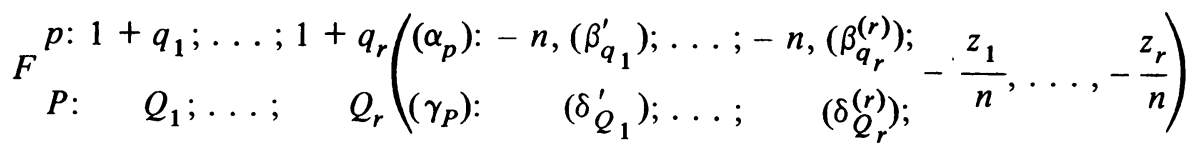

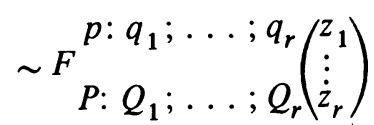

$$
\begin{aligned}
& -\frac{1}{2 n}\left[D_{2,0, \ldots, 0}\left(z_{1}, \ldots, z_{r}\right)+\cdots+D_{0, \ldots, 0,2}\left(z_{1}, \ldots, z_{r}\right)\right] \\
& +O\left(1 / n^{2}\right), \quad n \rightarrow \infty
\end{aligned}
$$

provided that (4.3) and (4.4), or (4.3) and (4.5), hold.

For special values of the nonnegative integers $p, q_{i}, P$ and $Q_{i}, i=1, \ldots, r$, (4.7) reduces to the corresponding asymptotic expansions for the Lauricella functions except possibly the function $F_{C}^{(r)}$ of the third type. In particular, for the fourth 
Lauricella function $F_{D}^{(r)}$, we have from (4.7) with $p=P=1, q_{i}=Q_{i}=0, i=1$, ..., $r$,

$$
\begin{aligned}
& F_{D}^{(r)}\left[\alpha,-n, \ldots,-n ; \gamma ;-z_{1} / n, \ldots,-z_{r} / n\right] \sim_{1} F_{1}\left[\alpha ; \gamma ; z_{1}+\cdots+z_{r}\right] \\
& \quad-\frac{\alpha(\alpha+1)\left(z_{1}^{2}+\cdots+z_{r}^{2}\right)}{2 n \gamma(\gamma+1)}{ }_{1} F_{1}\left[\alpha+2 ; \gamma+2 ; z_{1}+\cdots+z_{r}\right] \\
& \quad+O\left(n^{-2}\right), \quad n \rightarrow \infty
\end{aligned}
$$

where ${ }_{1} F_{1}[\alpha ; \gamma ; z]$ is the familiar confluent hypergeometric function.

Evidently, this last asymptotic expansion (4.8) would hold for all $z_{1}, \ldots, z_{r}$.

Department of Mathematics

University of Victoria

Victoria, B.C., Canada V8W 2Y2

Department of Mathematics

Ravenshaw College

Cut tack 3, Orissa, India

1. V. M. BHISE \& V. L. DESHPANDE, "On asymptotic confluent expansions for functions of two variables," Nederl. Akad. Wetensch. Proc. Ser. A, v. $75=$ Indag. Math., v. 34, 1972, pp. 106-112. MR 48 \#8863.

2. V. L. DESHPANDE, "Theorems involving confluent asymptotic expansions of functions of two variables," An. Univ. Timişoara Ser. Şti. Mat., v. 8, 1970, pp. 143-151. MR 48 \#4375.

3. V. L. DESHPANDE, "Confluent asymptotic expansions for functions of two variables," Mat. Vesnik, v. 10 (25), 1973, pp. 221-226. MR 49 \#640.

4. V. L. DESHPANDE, "Confluent expansions for functions of two variables," Math. Comp., v. 28, 1974, pp. 605-611. MR $49 \# 5409$.

5. JERRY L. FIELDS, “Confluent expansions,” Math. Comp., v. 21, 1967, pp. 189-197. MR 37 \#479.

6. YUDELL L. LUKE, The Special Functions and Their Approximations. Vol. I, Math. in Sci. and Engineering, vol. 53, Academic Press, New York and London, 1969. MR 39 \#3039.

7. H. M. SRIVASTAVA \& M. C. DAOUST, "Certain generalized Neumann expansions associated with the Kampé de Fériet function," Nederl. Akad. Wetensch. Proc. Ser. A, v. $72=$ Indag. Math., v. 31, 1969, pp. 449-457. MR $40 \# 5918$.

8. H. M. SRIVASTAVA \& M. C. DAOUST, "A note on the convergence of Kampé de Fériet's double hypergeometric series," Math. Nachr., v. 53, 1972, pp. 151-157. MR 47 \#511. 